\title{
Molecular Characterization and Overexpression of VpRPW8s from Vitis pseudoreticulata Enhances Resistance to Phytophthora capsici in Nicotiana benthamiana
}

\author{
Gongti Lai ${ }^{1,2}$, Peining Fu ${ }^{1,2}$, Yunxiao Liu ${ }^{1,2}$, Jiang Xiang ${ }^{2}$ and Jiang $\mathrm{Lu}^{2,3, *}$ \\ 1 College of Food Science and Nutritional Engineering, China Agricultural University, Beijing 100083, China; \\ laigt4769@cau.edu.cn (G.L.); fupeining@cau.edu.cn (P.F.); yunxiao_liu@cau.edu.cn (Y.L.) \\ 2 Center for Viticulture and Enology, School of Agriculture and Biology, Shanghai Jiao Tong University, \\ Shanghai 200240, China; xiangiang717@163.com \\ 3 Guangxi Crop Genetic Improvement and Biotechnology Laboratory, Guangxi Academy of Agricultural \\ Sciences, Nanning 530007, China \\ * Correspondence: jiang.lu@sjtu.edu.cn; Tel.: +86-021-3420-6005
}

Received: 24 January 2018; Accepted: 27 February 2018; Published: 13 March 2018

\begin{abstract}
R P W 8$ genes are atypical broad-spectrum genes that provide resistance to powdery mildew, downy mildew, the cauliflower mosaic virus in Arabidopsis thaliana, and powdery mildew in tobacco. They play important roles in basal plant pathogen defense. They also provide insights into a novel disease resistance mechanism. In this study, we report on homologous $R P W 8$ genes in Vitis pseudoreticulata. Five VpRPW8 genes were cloned; their Open Reading Frame (ORF) sequences ranged from 1994 base pairs to 2478 base pairs. They were comprised of five exons and four introns and shared $78.66 \%$ identity. Their proteins had typical conserved RPW8 and NB-LRR (the nucleotide-binding site and the leucine-rich repeats) domains (except VpRPW8-d, which lacked LRR domains). Prokaryotic expression results were consistent with predicted molecular weights. All five RPW8 genes were located in the cytoplasm. Quantitative real-time PCR (qRT-PCR) analysis showed that VpRPW8s in V. pseudoreticulata were induced by Plasmopara viticola, but nearly only $V v R P W 8-d$ genes were induced in Vitis vinifera. Furthermore, a VpRPW8 transgenic tobacco system was established. Overexpressed VpRPW8s enhanced resistance to Phytophthora capsici and VpRPW8s conferred varying degrees of resistance to Ph. capsici in Nicotiana benthamiana. Our study presents novel members of the plant RPW8 family and suggests that VpRPW8s are involved in enhanced resistance to P. viticola and Ph. capsici.
\end{abstract}

Keywords: Vitis pseudoreticulata; RPW8; downy mildew; Plasmopara viticola; Phytophthora capsici; overexpression

\section{Introduction}

Grapevine (Vitis vinifera) is the fourth flowering plant, the second woody plant, and the first fruit crop whose genome has been completely sequenced [1]. Grapevine contends with real disease threats such as downy mildew (DM), powdery mildew, and anthracnose. Downy mildew, caused by Plasmopara viticola $(\mathrm{Pv})$, is one of the most serious diseases in grapevines. P. viticola is responsible for substantial yield losses in grapevines. P. viticola is a biotrophic oomycete pathogen germinating from zoospores. Its hyphal tips penetrate stomatal openings and develop mycelia with haustoria inside the mesophyll tissue. A new round of infection occurs when sporangia form and release sporangiophores after the initial infection. P. viticola is an obligate grapevine parasite. It can severely damage $V$. vinifera leaves, young shoots, tendrils, and fruits [2-5]. However, there are many wild grapevine germplasms 
which are resistant to this disease in China [6-8]. V. pseudoreticulata, a Chinese endemic wild grapevine, is highly resistant to several diseases including downy mildew [9]. V. pseudoreticulata has colonized a wide range of habitats and soils, and harbors many predicted resistance genes ( $R$ genes). $R$ gene cloning, validation, and application have been important grapevine research objectives. Over the past several years, more than 13 loci resistant to $P$. viticola $(r p v)$ have been mapped on various chromosomes in Muscadinia rotundifolia, north American cultivars, and V. amurensis [10-17]. Only rpv1 was identified in a cross between $V$. vinifera 'Syrah' and M. rotundifolia "28-8-78" [10]. Nevertheless, there have been few studies on quantitative trait loci (qtl) or resistance genes in V. pseudoreticulata.

Plants and pathogens have evolved sophisticated relationships. Basal defense and $R$ gene-mediated defense are the two major types of disease resistance in plants [18]. Basal defense in plants includes innate immunity involved in pattern-triggered immunity (PTI) [19-21]. $R$ gene-mediated defense is plant-adaptive immunity involved in effector-triggered immunity (ETI) [22]. Once a pathogen secretes effectors to breach basal defense, plants activate $R$ genes that recognize the effectors and prevent pathogen invasion. In cloned $R$ genes, the nucleotide-binding site (NB) domains and the leucine-rich repeats (LRR) constitute the majority. In Arabidopsis thaliana, resistance to powdery mildew8 (RPW8) consists of RPW8.1 and RPW8.2 genes. These genes were first characterized to be resistant to many powdery mildew pathogens. As atypical $R$ genes, RPW8.1 and RPW8.2 had no NB or LRR domains but were able to induce localized, salicylic acid (SA)-dependent defenses [23]. When powdery mildew pathogens form haustoria to absorb plant nutrients, $R P W 8.2$ is induced and targets extra-haustorial membranes (EHM). Utilizing the SA signaling pathway, RPW8.2 induces haustorial complex formation and hydrogen peroxide $\left(\mathrm{H}_{2} \mathrm{O}_{2}\right)$ accumulation. Therefore, haustorial interception is the key to reducing oxidative damage in the host cell for RPW8-mediated mildew resistance [23-25].

Overexpressing RPW8 genes enhance resistance to powdery mildew, Hyaloperonospora parasitica and the cauliflower mosaic virus [26]. Studies have demonstrated that RPW8 genes enhance basal defense against biotrophic pathogens, which varies from powdery mildew-specific $R$ genes. Based on this hypothesis, we conducted experiments to verify the disease resistance functions of $V p R P W 8 s$. In this study, we isolated five RPW8 genes from $V$. pseudoreticulata and compared their transcriptional expression patterns with those in $V$. vinifera in response to $P$. viticola infection. We also assessed Phytophthora capsici resistance in transient transgenic tobacco overexpressing VpRPW8 genes. Finally, we generated stable transgenic tobacco lines overexpressing $V p R P W 8$ genes in order to elucidate $V p R P W 8 s$ resistance functions.

\section{Results}

\subsection{Isolation, Characterization, and Bioinformatics of VpRPW8s}

Five RPW8 genes were obtained from V. pseudoreticulata. These genes were named VpRPW8- $a$ (GenBank: KU365990), VpRPW8-b (GenBank: KU365991), VpRPW8-c (GenBank: KU365992), VpRPW8-d (GenBank: KX389173), and VpRPW8-e (GenBank: KX389175). RPW8-d had the shortest ORF (1994 base pairs) and RPW8- $a$ had the longest (2478 base pairs). The average ORF length was 2358 base pairs. The genes encoded proteins 647 to 825 amino acid residues long. The average protein product molecular weight was $89.11 \mathrm{kD}$. Multiple gene sequence alignments showed that they share an average of $78.66 \%$ identity. RPW8- $a$ and RPW8- $d$ shared the lowest identity (37.33\%) whereas RPW8-c and RPW8-e shared the highest identity (98.98\%) (see Supplementary Table S1). Gene structure analysis revealed that all five genes contained five exons and four introns (see Figure 1a). RPW8- $a$ and $R P W 8-b$ had long introns and shared the same gene structure model. The sizes of the four introns in RPW8-c, $R P W 8-d$, and RPW8-e were relatively shorter and more uniform. Therefore, RPW8-c, RPW8-d, and $R P W 8-e$ were grouped in another gene structure model. Additional information on VpRPW8s can be found in Supplementary Table S2. 
(a)

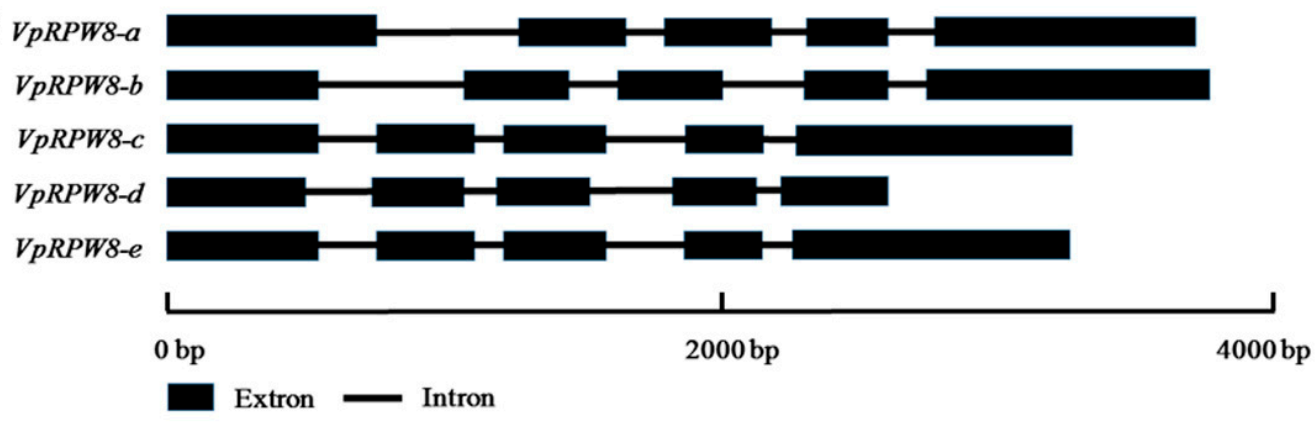

(b) Bright field Green fluorescence Merge

Bright field Green fluorescence Merge
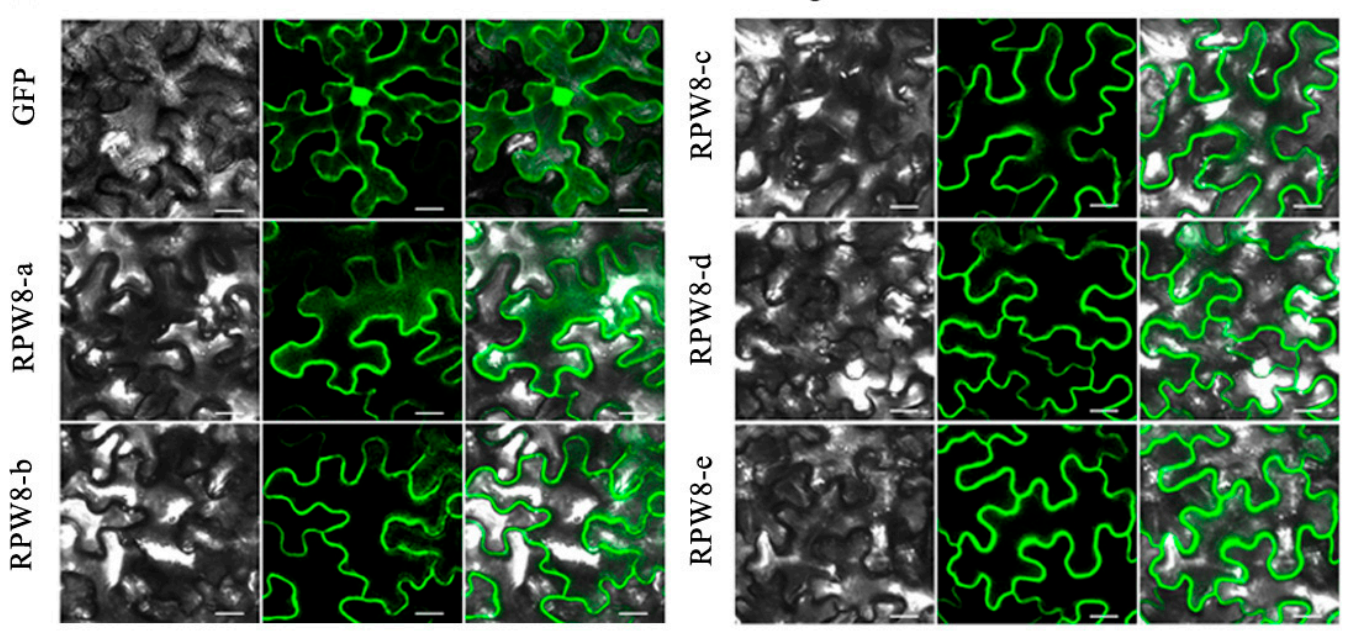

(c)

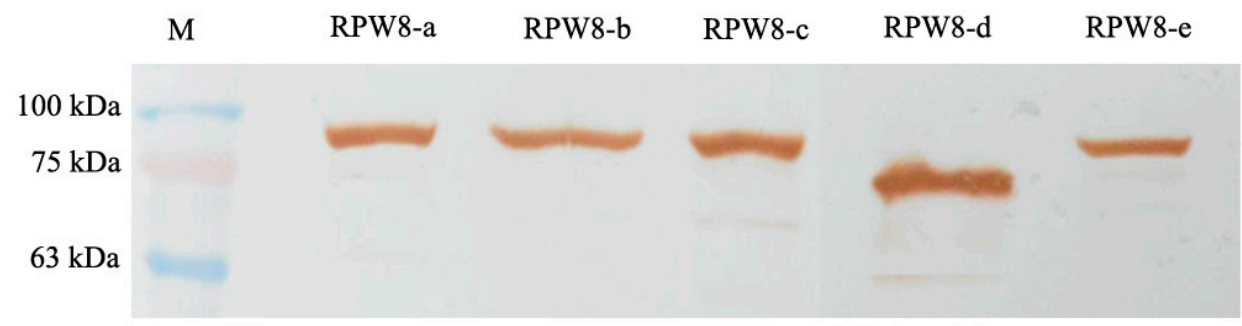

Figure 1. Gene structure, subcellular location, and prokaryotic expression of VpRPW8s. (a) Gene structure of $V p R P W 8 s$ consisted of five exons and four introns. They showed different gene length, but similar exon and intron structure. They can be grouped into two different gene structure models based on their different intron length. (b) Subcellular location of VpRPW8s. VpRPW8s were localized in the cytoplasm. Scale bar $=20 \mu \mathrm{m}$. (c) Prokaryotic expressions of VpRPW8s. VpRPW8-a, VpRPW8-b, VpRPW8-c, and VpRPW8-e were between 75 and $100 \mathrm{kD}$ whereas VpRPW8-d was $75 \mathrm{kD}$. The results of prokaryotic expression were consistent with predicted data.

Conserved domain predictions showed that they all had RPW8 and disease resistance domains like NB (nucleotide binding site) and LRR (leucine-rich repeat) (Figure 2). The exception was VpRPW8-d, which lacked LRR. VpRPW8-a had a long LRR motif starting from amino acid (AA) residue 183 and extending to $\sim$ AA residue 821 . The other LRR motifs started from $\sim$ AA residue 600 and extended to $\sim$ AA residue 800 . Previous studies showed that the RPW8 motif was the atypical $R$ gene domain and the NB-LRR motif was the typical $R$ gene domain. As a result, VpRPW8 proteins had both the atypical RPW8 broad-spectrum structure and the typical $R$ gene structure. Therefore, the $V p R P W 8 s$ may be defined as functional disease resistance genes. 

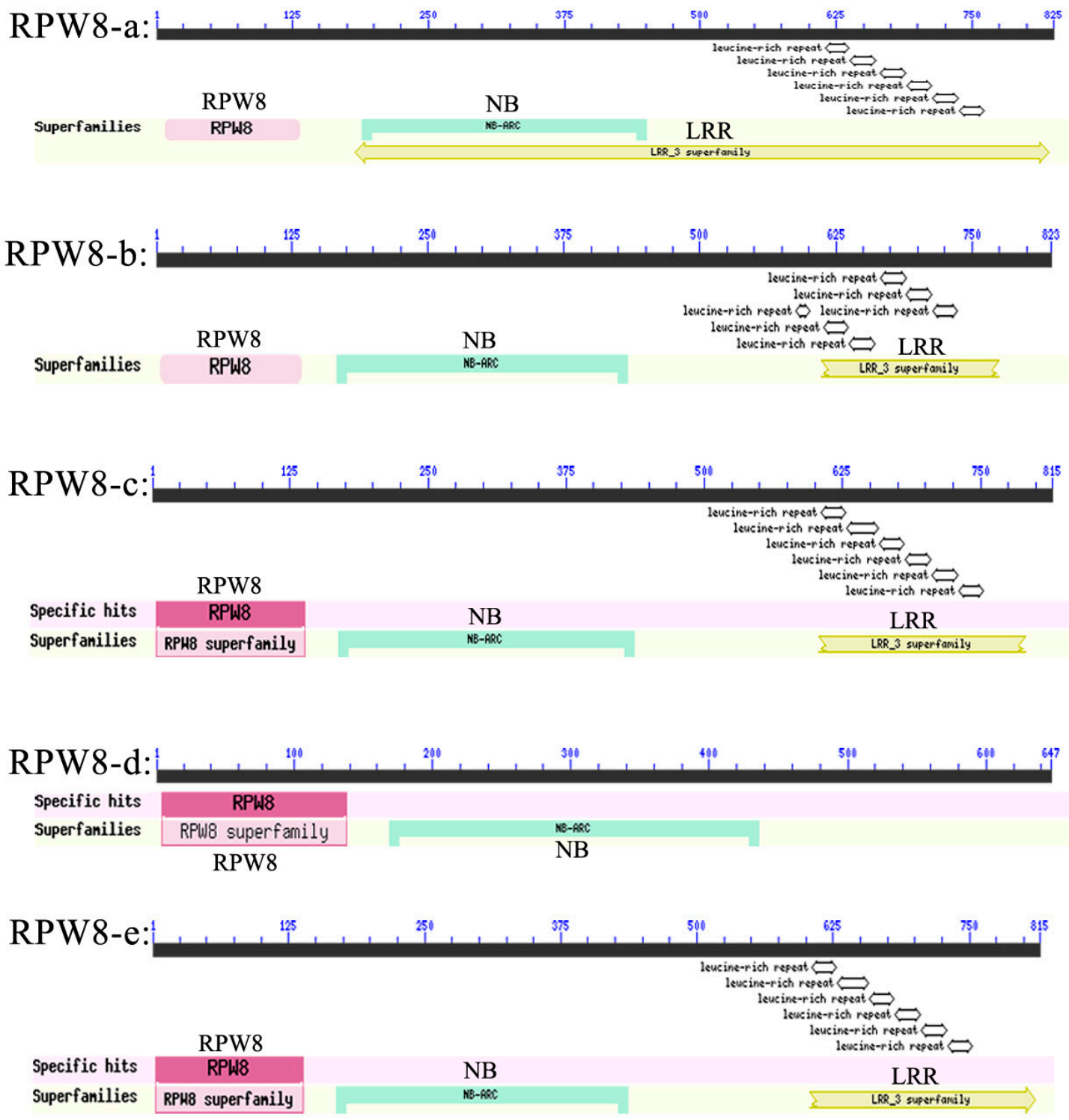

Figure 2. Conserved domains analysis of VpRPW8s. RPW8s contained RPW8 domains, conserved disease resistance domains NB (nucleotide binding sites) and LRR (leucine-rich repeats) except for VpRPW8-d. VpRPW8-d had the RPW8 and NB domains but not LRR. VpRPW8-a had a long LRRs motif starting from $\sim$ AA residue 183 and extending to $\sim$ AA residue 821 . The other LRR motifs started from $\sim$ AA residue 600 and extended to $\sim$ AA residue 800 .

\subsection{Transcriptional Expression Analysis of the RPW8s in V. pseudoreticulata and V. vinifera under} P. viticola Stress

$V$. pseudoreticulata has resistant compounds whereas $V$. vinifera is susceptible. Nevertheless, little is known about the $R$ genes in grapevines or how they work. Whether the RPW8s play important roles in P. viticola infection remains unknown. In this study, the transcriptional expression levels of the five $R P W 8$ genes were determined under $P$. viticola stress. The results showed that the RPW8s expressed differently in $V$. pseudoreticulata and $V$. vinifera (see Figure 3). The RPW8 genes were abundantly upregulated in $V$. pseudoreticulata but their expression levels varied somewhat in $V$. vinifera. In $V$. vinifera, the expression levels were lower than those for the control $(0 \mathrm{hpi})$ in all treatments except for $V v R P W 8-d$ (all treatments) and $V v R P W 8-c(24 \mathrm{hpi})$. VvRPW8-d genes were upregulated and had high expression levels at 6 and 48 hpi. The transcription levels of $V p R P W 8 s$ changed significantly in 
the early stages of pathogenesis, then reached a maximum, and gradually decreased. The exception was RPW8-a, which was not dramatic upregulated. Taken together, all VpRPW8s were induced by $P$. viticola in $V$. pseudoreticulata, rather than $V v R P W 8 s$. Therefore, $V p R P W 8 s$ participated in downy mildew resistance rather than $V v R P W 8$ s.

(a)

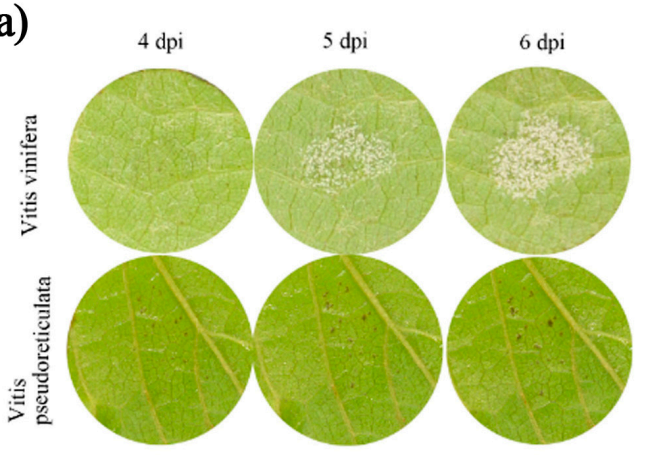

(c)

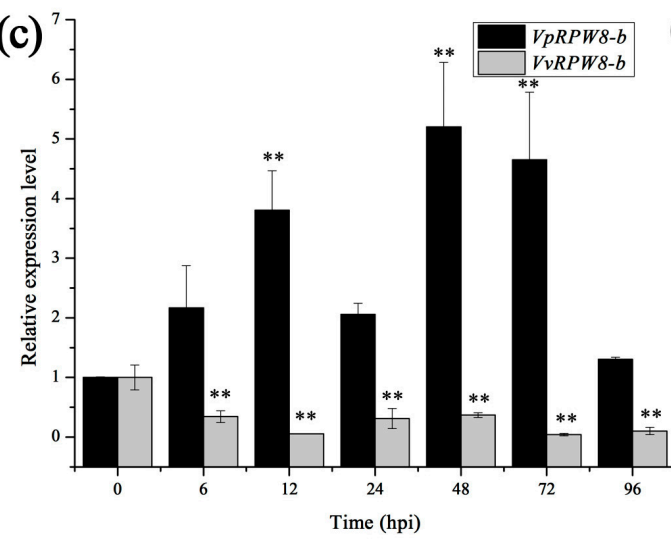

(e)

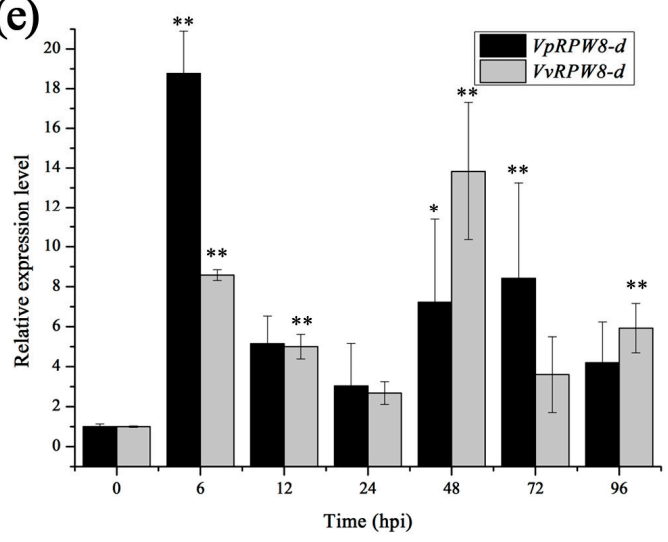

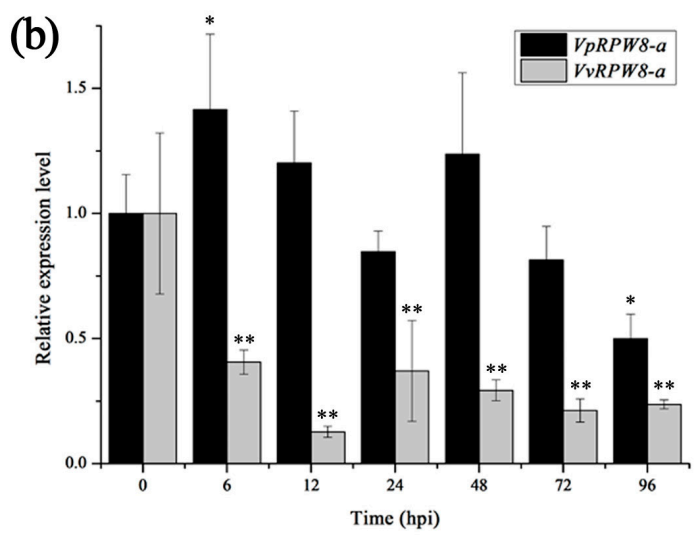

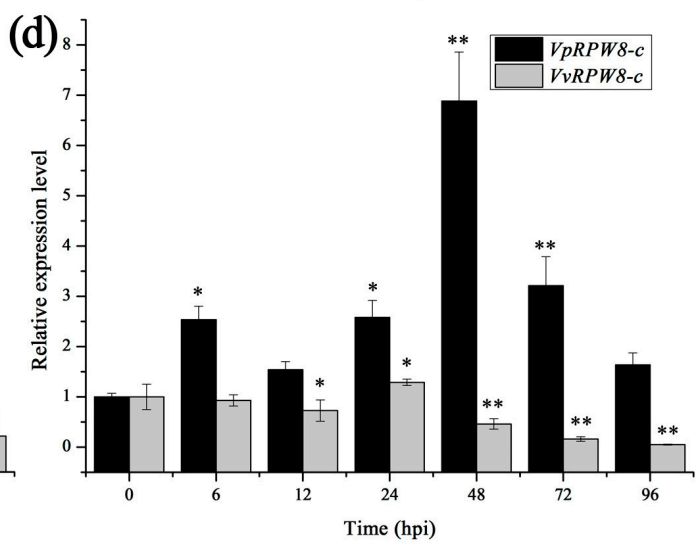

(f)

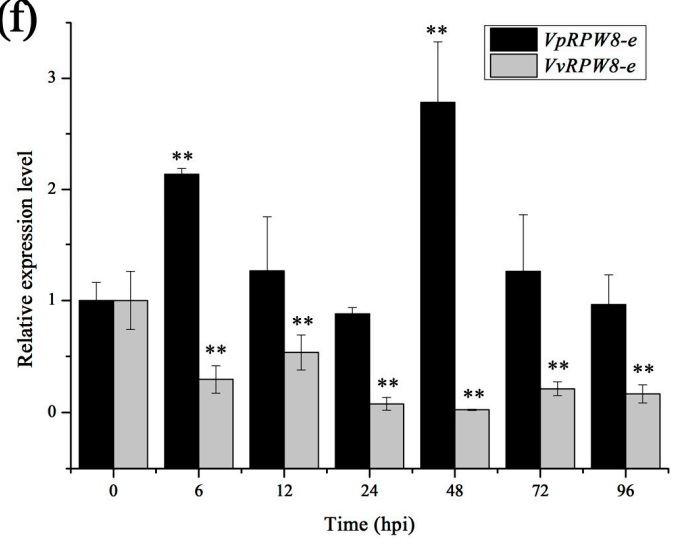

Figure 3. Transcriptional expression of RPW8s in V. pseudoreticulata and V. vinifera in response to $P$. viticola. (a) The disease symptom of $V$. vinifera and $V$. pseudoreticulata under P. viticola infection. (b-f) Transcriptional expression of RPW8s in V. pseudoreticulata and V. vinifera in response to P. viticola. $R P W 8$ genes were significantly upregulated in $V$. pseudoreticulata but downregulated in $V$. vinifera (RPW8-a, b, e). In $V$. vinifera, the expression levels were lower than those for the control (0 hpi) in all treatments except for $V v R P W 8-d$ (all treatments) and $V v R P W 8-c$ (24 hpi). VvRPW8-d genes were upregulated and had high expression levels at 6 and 48 hpi. The transcriptional levels of $V p R P W 8 \mathrm{~s}$ changed significantly in the early stages of pathogenesis, reached a maximum, and then gradually decreased. The exception was $V p R P W 8-a$, which was not significantly upregulated (except for 6 hpi, ** $p<0.01, * p<0.05)$. 
Two different expression patterns were detected. The first was early ( $6 \mathrm{hpi}$ ) induction followed by sustained high expression levels at $48 \mathrm{hpi}$. This pattern applied to VpRPW8-a, VpRPW8-d, and $V p R P W 8-e$. In the second pattern, induction gradually increased and reached a maximum at $48 \mathrm{hpi}$. Such was the case for VpRPW8- $b$ and VpRPW8-c. A wide range of expression efficiency was observed for $V p R P W 8 s$. VpRPW8- $d$ genes were the most strongly induced and had an $18.7 \times$ expression level at 6 hpi. The expression levels of $V p R P W 8-b$ and $V p R P W 8-c$ peaked at 48 hpi with $5.2 \times$ and $6.8 \times$, respectively. $V p R P W 8-a$ and $V p R P W 8-e$ genes were expressed at far lower levels, which were $1.4 \times(6 \mathrm{hpi})$ and $2.8 \times(48 \mathrm{hpi})$, respectively. Therefore, VpRPW8s (RPW8- $d$ and $R P W 8-c$ as well as $R P W 8-b, R P W 8-e$, and $R P W 8-a$ in sequence) play key roles in response to $P$. viticola infection in $V$. pseudoreticulata. In contrast $V v R P W 8 s$ (except $V v R P W 8-d$ ) may not be actively involved in the defense against $P$. viticola in $V$. vinifera.

\subsection{Prokaryotic Expression and Subcellular Localization of VpRPW8s}

In this study, $p E T 30 a-R P W 8 s-H i s$ were constructed and transformed into E. coli BL21. Prokaryotic $V p R P W 8 s$ expressions were examined by using Western blot. The results indicated that the VpRPW8-a, VpRPW8-b, VpRPW8-c, and VpRPW8-e proteins ranged in size from 75 to $100 \mathrm{kD}$. VpRPW8-d was $\sim 75 \mathrm{kD}$ (see Figure 1c). A bioinformatics analysis showed that the RPW8 proteins were 93.92, 93.04, $92.45,73.62$, and $92.52 \mathrm{kD}$, respectively. Therefore, the prokaryotic expression was consistent with predicted data and the $V p R P W 8 s$ expressed their protein products as expected.

To determine the subcellular locations of the VpRPW8s, $p B$ I121-RPW8s-GFP were constructed and transformed into Agrobacterium tumefaciens. The A. tumefaciens suspensions were injected into Nicotiana benthamiana leaves to express the RPW8s-GFP fusion proteins. GFP fluorescent signals for the five $R P W 8$ genes were observed by using confocal microscopy and found mainly in the cytoplasm (see Figure 1b), which suggests the cytoplasmic location for these five $V p R P W 8$ genes.

\subsection{Transiently Expressing RPW8s Enhanced Resistance to Ph. capsici}

To determine whether VpRPW8s enhance Ph. capsici resistance in N. benthamiana, transiently transgenic tobacco leaves were inoculated with Ph. capsici. As shown in Figure 4a, there were signs of necrosis in the tobacco leaves at $36 \mathrm{hpi}$. Necrosis was more severe in the controls on the right side of the leaves than the experimental group expressing VpRPW8s on the left side of the leaves. At 54 hpi, the necrotic areas expanded and the difference between the control and the experimental groups was more significant.

The resistance to $P h$. capsici induced by transient $R P W 8$ s expression was investigated by calculating the areas of leaf necrosis in $N$. benthamiana. The ratio of control to transgenic leaf necrotic areas was defined as the relative disease index. The relative disease index increased when disease symptoms grew. Statistical analysis showed that the relative disease index of controls was significantly higher than in $V p R P W 8$ transgenic groups (see Figure $4 \mathrm{~b}, \mathrm{c}$ ). Therefore, $V p R P W 8 s$ enhanced $P h$. capsici resistance in transiently transformed $N$. benthamiana. 

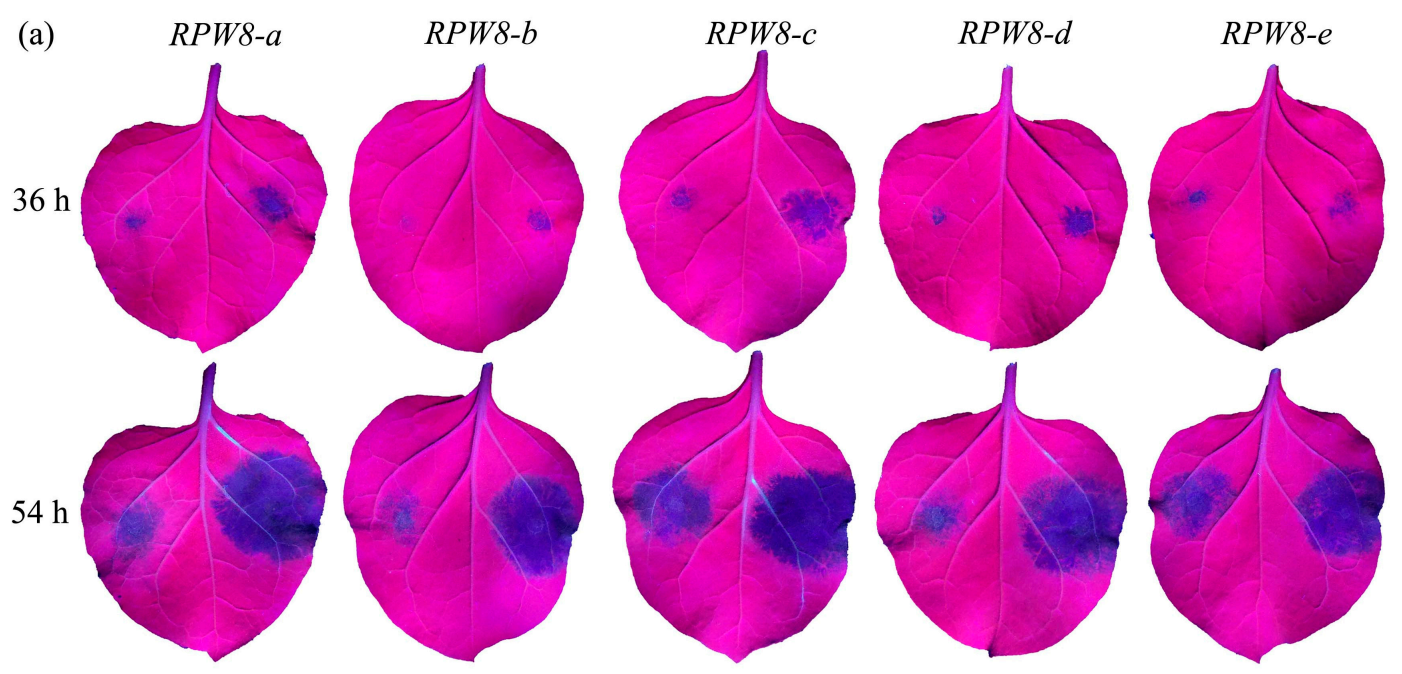

(b)
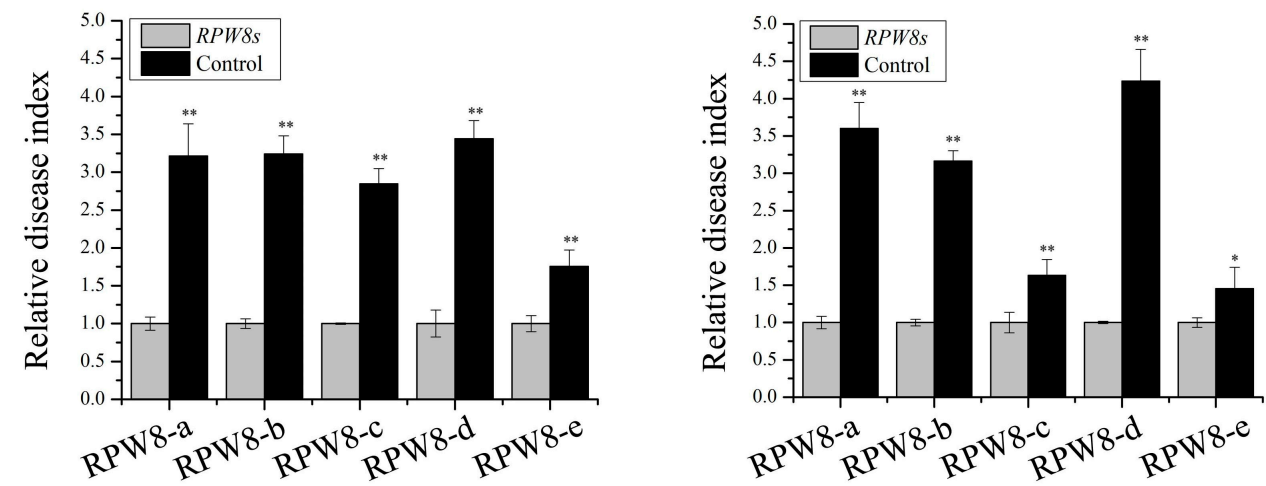

Figure 4. Transiently expressing $V p R P W 8$ s enhanced $P h$. capsici resistance in $N$. benthamiana. (a) Lesions on the N. benthamiana leaves transiently expressing VpRPW8s at $36 \mathrm{hpi}$ and 54 hpi. Symptoms on the right side of the leaves represent the control while those on the left side represent transiently expressing VpRPW8s. The necrotic areas expanded over time (36-54 hpi). The experimental group showed somewhat smaller necrotic areas. (b) Disease indices for N. benthamiana at 36 hpi. (c) Disease indices for N. benthamiana at $54 \mathrm{hpi}$. Disease index (Y-axis) is the ratio of the control necrotic areas to those on the transgenic plants $\left({ }^{* *} p<0.01,{ }^{*} p<0.05\right)$.

\subsection{Stably Expressing RPW8s Enhanced Resistance to Ph. capsici}

The aforementioned experiments revealed specific RPW8s structures and demonstrated their disease resistance functions. Based on the results of the transient $V p R P W 8$ s transformation experiment, stable transformations were conducted. All five transgenic lines were obtained by kanamycin resistance selection (T1) and by using DNA-PCR detection (T1). More than Forty-five T2 seedlings were then germinated from T1 seeds in kanamycin resistance medium. Transcriptional analysis of these T2 lines indicated that more than 45 plants or all of the plants studied overexpressed RPW8s (see Figure 5c).

To verify Ph. capsici resistance, we inoculated detached leaves and living plants with the pathogen (see Figure 5a). Necrotic areas (relative disease index) on the detached leaves in order of increasing size were labeled RPW8-d, RPW8- $a$, RPW8-b, RPW8-e, RPW8-c, and CK (WT and pBI121-GFP in Figure 5d). These results somewhat corroborated the data obtained for the transient transformation experiment. This showed the transgenic group was more resistant to Ph. capsici than the control groups. 

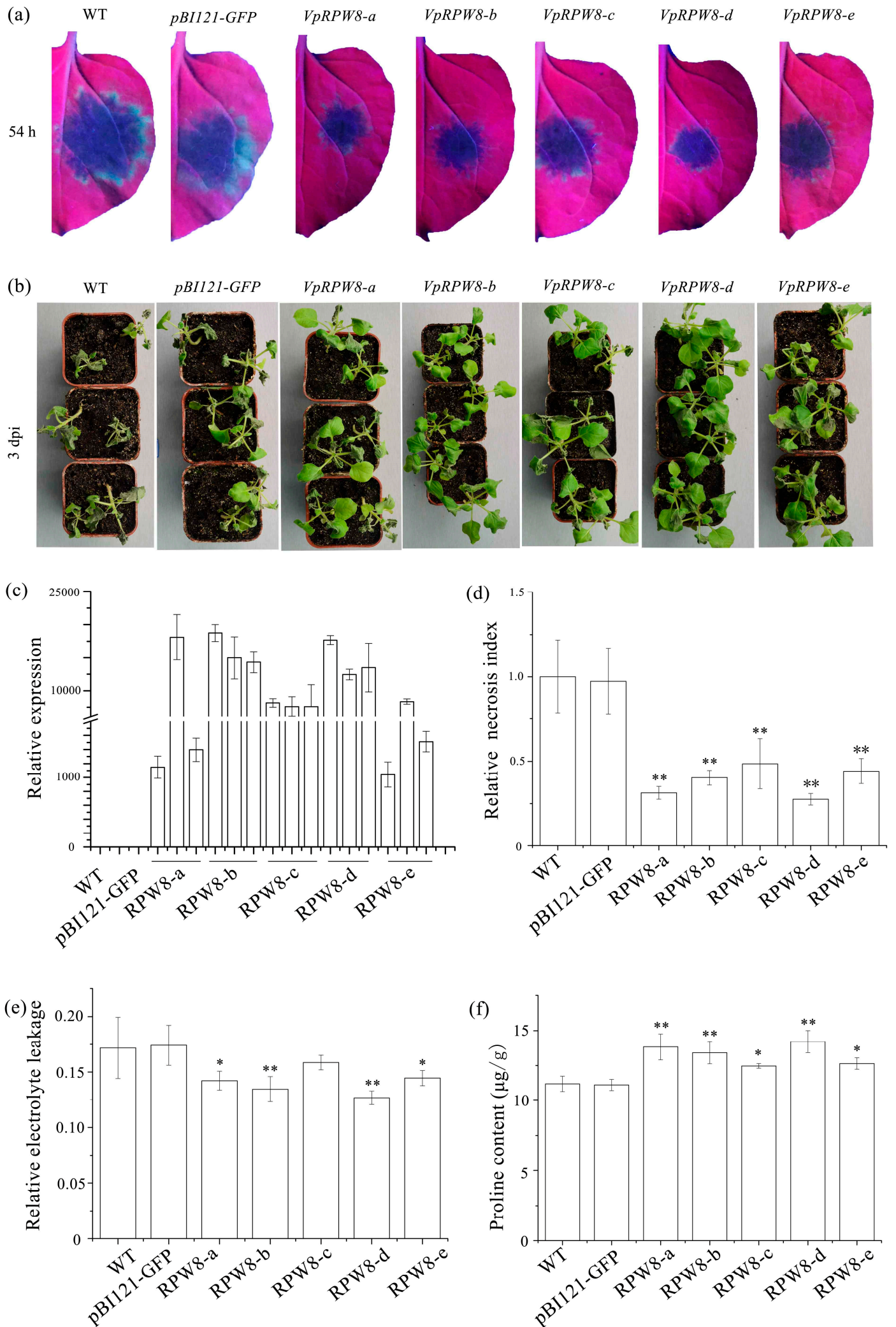

Figure 5. $V p R P W 8 s$ enhanced $P h$. capsici resistance in transgenic $N$. benthamiana. (a) Lesions on detached $N$. benthamiana leaves expressing VpRPW8s. (b) Disease symptoms in whole N. benthamiana plants expressing VpRPW8s. (c) Transcriptional expression of $R P W 8 \mathrm{~s}$ in transgenic N. benthamiana (T2). (d) Relative disease index on detached N. benthamiana leaves expressing VpRPW8s. (e) Relative electrolyte leakage in whole $N$. benthamiana plants expressing VpRPW8s. (f) Proline content in whole $N$. benthamiana plants expressing VpRPW8s $\left({ }^{* *} p<0.01,{ }^{*} p<0.05\right)$. 
Both the control and transgenic plants displayed varying degrees of disease symptoms. As shown in Figure $5 b$, the control plants were severely wilted while a few turgid leaves remained in the plants of the experimental group. Since the previous experiments indicated that $P$. viticola significantly induced $V p R P W 8$ s at $48 \mathrm{hpi}$, we measured relative electrolyte leakage and proline content at $48 \mathrm{hpi}$ to determine the disease resistance response. The relative electrolyte leakage in the VpRPW8 transgenic plants was lower than that in the control plants (see Figure 5e). In contrast, the proline content in the VPRPW8 transgenic plants was higher than that in control plants (see Figure $5 \mathrm{f}$ ). These findings indicate that $R P W 8$ s enhanced Ph. capsici resistance in N. benthamiana.

The relative electrolyte leakages in order of increasing magnitude were labeled as RPW8- $d$, RPW8- $a$, RPW8-b, RPW8-e, RPW8-c and CK (WT and $p B I 121-G F P$ ). The proline contents in order of decreasing magnitude were labeled as RPW8-d, RPW8- $a, R P W 8-b, R P W 8-e, R P W 8-c$, and CK (WT and $p B I 121-G F P)$. Therefore, $N$. benthamiana plants expressing $V p R P W 8 s$ were more resistant to $P h$. capsici than CK plants. VpRPW8- $d$ genes were deduced to be the most resistant followed by VpRPW8- , $V p R P W 8-b, V p R P W 8-e$ and $V p R P W 8-c$ in sequence.

\section{Discussion}

\subsection{Conserved Domains and Sequence Polymorphism of RPW8s}

In plants, the RPW8 genes constitute a superfamily. They were first reported in A. thaliana in 2001 [23]. It was already known that the $R P W 8 s$ had an atypical $R$ gene structure but the same mechanism as $R$ genes. All RPW8s share the conserved RPW8 domains. The predicted AtRPW8.1 and AtRPW8.2 genes have $45.2 \%$ sequence identity [23]. In Brassica spp., they are highly homologous and their sequence identity ranges from $90 \%$ to $95 \%$ [27,28]. In the present study, we predicted the typical R gene domain NB-LRR as well as the conserved RPW8 domains. Multiple sequence alignments of RPW8 genes showed $78.66 \%$ nucleotide and $74.28 \%$ protein identity. This seemed an apparently high identity, but pairwise sequence alignment revealed that the nucleotide sequence identity ranged from $37.33 \%$ (protein, $25.45 \%$ ) to $98.98 \%$ (protein, $97.67 \%$ ) (see Supplementary Table S1).

The AtRPW8.1 gene was localized in the mesophyll cells and was surrounded by punctuated spots proximal to the chloroplasts. In contrast, the AtRPW8.2 gene was observed in both the epidermal and mesophyll cells. AtRPW8.1 and AtRPW8.2 genes were heterogeneous and may have spatially differed from each other. Nevertheless, they were both resistant to biotrophic pathogens $[25,26]$. In $V$. pseudoreticulata, RPW8s genes were localized mainly in the cytoplasm. Plant RPW8s are also diverse in terms of subcellular location. Therefore, $R P W 8$ s have conserved structures while also having sequence and functional polymorphism.

\subsection{VpRPW8s Participate in Resistance to P. viticola}

Previous studies indicated that $V$. vinifera was generally susceptible to downy mildew caused by P. viticola. In recent years, Rpv1, NPR1 homologs, and PR protein-encoding genes have been identified as downy mildew resistance-associated genes $[10,29,30]$. In our previous study, we presented a series of candidate genes that may contribute to downy mildew resistance [31]. To date, however, little is known about $R$ genes, and obstacles persist in $V$. vinifera breeding improvements. In contrast, the Chinese wild grape $V$. pseudoreticulata showed strong resistance to downy mildew. For this reason, $V$. pseudoreticulata was used as a germplasm to breed disease resistance and has proven to be an invaluable entity in functional genomics. In our study, $R P W 8$ s are differentially expressed in $V$. vinifera and V. pseudoreticulata in response to P. viticola. VpRPW8 genes were strongly induced by P. viticola, which first rose and then fell in expression levels over time. Two different expression patterns were observed for VpRPW8 genes. In the first pattern, VpRPW8- $a$, VpRPW8-d, and VpRPW8-e were involved, which indicates that $R P W 8 s$ were induced early and remained at a high expression level during the middle stage. In the second pattern, $V p R P W 8-b$ and $V p R P W 8-c$ were significantly induced and reached a peak during the middle stage. On the other hand, the expression levels of $V v R P W 8 s$ (except 
$V v R P W 8-d$ ) remained low and did not respond strongly to $P$. viticola. The expressions of the VpRPW8 genes resembled those for the NB-LRR $R$ genes expressed at low levels prior to pathogen invasion, but were then rapidly induced during the early stage of infection [32-35]. Therefore, $V p R P W 8 s$ are able to resist $P$. viticola in $V$. pseudoreticulata.

\subsection{Broad-Spectrum Disease Resistance of RPW8}

A study of the range of pathogens controlled by RPW8 in A. thaliana showed that transgenic plants were resistant to all powdery mildew pathogens tested [23]. A. thaliana expressing RPW8 genes also had enhanced resistance to Hyaloperonospora parasitica and even appeared to be resistant to the cauliflower mosaic virus when expressing RPW8s from their native promoters [26]. AtRPW8.1 and AtRPW8.2 genes also induced resistance to powdery mildews in tobacco [36]. In our study, $P$. viticola induced $V p R P W 8 s$ in $V$. pseudoreticulata. VpRPW8s played an important role in the response to $P$. viticola invasion. To examine $V p R P W 8 s$ disease resistance further, we established a $V p R P W 8 s$ overexpression system in N. benthamiana using Agrobacterium-mediated transformation. The excised leaves from both transiently and stably transformed transgenic plants infected with Ph. capsici had smaller necrotic areas than those of either the wild type or the control plants. Whole tobacco plants overexpressing $V p R P W 8$ genes appeared to be more resistant to Ph. capsici than either the wild type or the control. $V p R P W 8$ genes also enhanced resistance to Phytophthora parasitica in our other experiments. In summary, $V p R P W 8 s$ enhanced resistance to $P$. viticola, Ph. capsici, and Ph. parasitica. Therefore, $V p R P W 8 s$ are broad-spectrum disease resistant genes.

\section{Materials and Methods}

\subsection{Plant Materials and Treatments}

One-year-old V. pseudoreticulata "1058" and V. vinifera "Cabernet Sauvignon" were grown in a greenhouse at $25 \pm 2{ }^{\circ} \mathrm{C}$ under a $16 \mathrm{~h} \mathrm{light} / 8 \mathrm{~h}$ dark photoperiod. For quantitative real-time PCR, grapevine leaves were inoculated with $10 \mu \mathrm{L}$ of an aqueous suspension of pathogen sporangia ( $10^{5}$ sporangia $\mathrm{mL}^{-1}$ ) and sampled at 0 hpi (hours post-inoculation), 6, 12, 24, 48, 72, and $96 \mathrm{hpi}$. The excised leaves were stored at $-80^{\circ} \mathrm{C}$. One-month-old $N$. benthamiana seedlings in vivo were used for transient transformation and disease resistance evaluation. The third to fifth unfolded leaves from the shoot apex were excised for inoculation with Ph. capsici. One-month-old N. benthamiana seedlings in vitro were used for stable genetic transformation with VpRPW8s. Transgenic N. benthamiana seedlings were transplanted to pots in the greenhouse when they were one month old (4 to 6-leaf stage).

\subsection{Gene Cloning and Sequence Analysis}

Total RNA was isolated from grapevine leaves according to a slightly modified cetyltrimethylammonium bromide (CTAB) method. The total RNA was used as a template for reverse transcription with a RevertAid TM First Strand cDNA Synthesis Kit (Invitrogen, Carlsbad, CA, USA). Primers for gene cloning were designed according to the five predicted $R P W 8$ sequences in Phytozome (https:/ / phytozome.jgi.doe.gov/pz/portal.html\#!search?show=KEYWORD) and PRG (http:/ / prgdb.crg.eu/wiki/Main_Page). Primer data is listed in Supplementary Table S3. The PCR products were sub-cloned in E. coli and sequenced. Bioinformatics analysis of the five RPW8s was performed using DNAMAN, ProtParam tool (http://web.expasy.org/protparam/), and NCBI-CDD (http:/ /www.ncbi.nlm.nih.gov/Structure/cdd/wrpsb.cgi).

\subsection{Quantitative Real-Time PCR Analysis}

To determine the transcription levels of the five RPW8s during the infections of $V$. pseudoreticulata and $V$. vinifera with $P$. viticola, leaves were excised and used for RNA extraction. The qRT-PCR was performed on the QIAGEN Rotor-Gene Q system (QIAGEN, Hilden, Germany) using a SuperReal PreMix Plus (SYBR Green) kit (TIANGEN Biotech Co., Ltd., Beijing, China). To calculate the 
transcription levels, constitutively expressed elongation factor1- $\alpha(E F 1-\alpha)$ was used as an internal gene for normalization [37] with the $2^{-\Delta \Delta C t}$ method [38]. All reactions were performed in triplicate.

\subsection{Prokaryotic Expression and Subcellular Localization Analysis}

For prokaryotic expression, the RPW8s PCR fragments were sub-cloned into $p E T 30 a$ to generate pET30a-RPW8-a, pET30a-RPW8-b, pET30a-RPW8-c, pET30a-RPW8-d, and pET30a-RPW8-e. The recombinant plasmids were then chemically transformed into E. coli BL21 which was grown in Luria-Bertani (LB) broth supplemented with kanamycin until the $\mathrm{OD}_{600}$ reached 0.4 . Then $10 \mathrm{mmol} \mathrm{L}^{-1}$ isopropyl $\beta$-D-1-thiogalactopyranoside (IPTG) was added to induce target protein expression. The proteins were verified by Western blot and dyed with $3^{\prime}$-diaminobenzidine (DAB).

For subcellular localization, $p B$ I121-RPW8-a-GFP, $p B$ I121-RPW8-b-GFP, $p B$ I121-RPW8-c-GFP, pBI121-RPW8- $d-G F P$, and $p B I 121-R P W 8-e-G F P$ were constructed using a $p B I 121-G F P$ plasmid (CaMV $35 S$ promoter-driven). The recombinant plasmids were transferred via electroporation into the Agrobacterium tumefaciens strain GV3101. The GV3101 containing these vectors was harvested by using centrifugation when the $\mathrm{OD}_{600}$ reached $\sim 1.0$ and resuspended in $10 \mathrm{mM} \mathrm{MgCl}_{2}$ to a final $\mathrm{OD}_{600}$ of 0.6. The transient transformations were performed according to a protocol previously described for N. benthamiana [39]. Fluorescence was visualized at $488 \mathrm{~nm}$ with an Olympus FluoView ${ }^{\mathrm{TM}}$ FV1000 (Olympus Corporation, Shinjuku, Tokyo, Japan).

\subsection{Transient Transformation and Resistance to Ph. capsici in N. benthamiana}

To determine RPW8s resistance to Ph. capsici, the aforementioned A. tumefaciens harboring pBI121-RPW8s-GFP were used to transform $N$. benthamiana. A $10 \mathrm{mM} \mathrm{MgCl} 2$ solution was prepared for transient transformation. Leaves were excised $48 \mathrm{~h}$ after being injected with the $A$. tumefaciens suspensions. A. tumefaciens harboring $p B I 121-R P W 8 s-G F P$ was injected on the left side of the back of the leaf while $p B I 121-G F P$ was injected on the right side as a type of control. $N$. benthamiana leaves were inoculated with $\mathrm{Ph}$. capsici pathogen disks cultured on solid oat medium. Leaf lesions were photographed with UV imaging at 36 and 54 hpi. Disease index was analyzed according to the ratio of the control necrotic areas to those on the transgenic plants.

\subsection{Stable Transformation and Resistance to Ph. capsici in N. benthamiana}

One-month-old in vitro $N$. benthamiana seedlings were used for genetic modification. The genetic manipulations were conducted as previously described, with minor modifications [40]. The bacterial strain and expressing vectors ( $p B I 121-R P W 8 s-G F P$ ) were described as transient expression, which was previously mentioned. To verify the transgenic $N$. benthamiana plants, kanamycin $\left(60 \mathrm{mg} \mathrm{L}^{-1}\right)$-resistant selection and DNA-PCR were performed on T1 plants. Kanamycin-resistant selection and transcription level analysis were performed on T2 plants as well. The T2 plants were used to evaluate VpRPW8s disease resistance. Excised-leaf inoculation with Ph. capsici was performed as described above for the transient transformation. For the whole-plant inoculation, $P$ h. capsici zoospore suspensions were prepared and adjusted to 100 zoospores $\mathrm{mL}^{-1}$ [41]. VpRPW8 plants were sprayed with zoospore suspensions. N. benthamiana wild type- and $p B$ I121-GFP transgenic plants were used as controls. All plants were maintained at $25 \pm 2{ }^{\circ} \mathrm{C}$. The chambers were sealed with plastic film to retain moisture. Relative electrolyte leakage [42] and proline content [43] were measured as previously described.

\section{Conclusions}

In this study, we cloned five $V p R P W 8$ genes from $V$. pseudoreticulata which is known to be highly resistant to downy mildew. These five genes harbor the same functional conserved domains as RPW8 and NB-LRR genes except for the VpRPW8- $d$ gene. All five $V p R P W 8$ genes consisted of five exons and four introns. Subcellular location showed they were localized in the cytoplasm. VpRPW8s were strongly induced by $P$. viticola in $V$. pseudoreticulata, rather than VvRPW8s.Therefore, VpRPW8 genes participate in downy mildew resistance. $V p R P W 8-d$ was the most highly expressed gene, followed by 
VpRPW8-b and VpRPW8-c. VpRPW8- $a$ and VpRPW8-e genes were expressed at low levels. Transient and stable transformations were carried out on $N$. benthamiana to observe the disease resistance functions of $R P W 8 s$. The results revealed that $V p R P W 8$ transgenic tobacco was highly resistant to $P h$. capsici; VpRPW8-d was deduced to be the most resistant gene. Taken together, we verified the novel members of the plant $R P W 8$ family and enriched the $\mathrm{R}$ gene associated with the research in grapevines. $V p R P W 8$ genes possess conserved domains, and sequence and function polymorphism. These are also considered broad-spectrum disease genes. The study of broad-spectrum-like resistance genes lays the foundation for future research and application in grapevine variety improvement.

Supplementary Materials: The following are available online at www.mdpi.com/1422-0067/19/3/839/s1.

Acknowledgments: This work was supported by National Natural Science Foundation of China (grant No. 31471754), China Agriculture Research System (grant no. CARS-30-yz-2).

Author Contributions: Jiang $\mathrm{Lu}$ and Gongti Lai designed the experiments. Gongti Lai, Yunxiao Liu, and Jiang Xiang prepared the materials. Gongti Lai and Peining Fu performed the experiments. Gongti Lai and Jiang Lu wrote and revised the manuscript. All the authors approved the final manuscript.

Conflicts of Interest: The authors declare no conflict of interest.

\section{Abbreviations}

$\begin{array}{ll}\text { RPW8 } & \text { Resistance to Powdery Mildew8 } \\ \text { R gene } & \text { Resistance Gene } \\ \text { NCBI } & \text { National Center for Biotechnology Information Search database } \\ \text { qRT-PCR } & \text { Quantitative Real-Time PCR } \\ \text { GFP } & \text { Green Fluorescent Protein } \\ \text { ORF } & \text { Open Reading Frame } \\ \text { hpi } & \text { Hours Post-Inoculation } \\ \text { dpi } & \text { Days Post-Inoculation }\end{array}$

\section{References}

1. Jaillon, O.; Aury, J.M.; Noel, B.; Policriti, A.; Clepet, C.; Casagrande, A.; Choisne, N.; Aubourg, S.; Vitulo, N.; Jubin, C.; et al. The grapevine genome sequence suggests ancestral hexaploidization in major angiosperm phyla. Nature 2007, 449, 463-467. [CrossRef] [PubMed]

2. Pearson, R.C.; Goheen, A.C. Compendium of Grape Diseases; APS Press: St. Paul, MO, USA, 1988, ISBN 0890540888.

3. Yu, Y.; Zhang, Y.; Yin, L.; Lu, J. The mode of host resistance to Plasmopara viticola infection of grapevines. Phytopathology 2012, 102, 1094-1101. [CrossRef] [PubMed]

4. Gessler, C.; Pertot, I.; Perazzolli, M. Plasmopara viticola: A review of knowledge on downy mildew of grapevine and effective disease management. Phytopathol. Mediter. 2011, 50, 3-44. [CrossRef]

5. Kiefer, B.; Riemann, M.; Büche, C.; Kassemeyer, H.H.; Nick, P. The host guides morphogenesis and stomatal targeting in the grapevine pathogen Plasmopara viticola. Planta 2002, 215, 387-393. [CrossRef] [PubMed]

6. Wang, L.; Wei, J.; Zou, Y.; Xu, K.; Wang, Y.; Lu, C.; Xu, Y. Molecular characteristics and biochemical functions of VpPR10s from Vitis pseudoreticulata associated with biotic- and abiotic stresses. Int. J. Mol. Sci. 2014, 15, 19162-19182. [CrossRef] [PubMed]

7. Wang, Y.; Liu, Y.; He, P.; Chen, J.; Lamikanra, O.; Lu, J. Evaluation of foliar resistance to Uncinula necator in Chinese wild Vitis species. Vitis 1995, 34, 159-164. [CrossRef]

8. Xu, W.; Yu, Y.; Zhou, Q.; Ding, J.H.; Dai, L.M.; Xie, X.Q.; Xu, Y.; Zhang, C.H.; Wang, Y.J. Expression pattern, genomic structure, and promoter analysis of the gene encoding stilbene synthase from Chinese wild Vitis pseudoreticulata. J. Exp. Bot. 2011, 62, 2745-2761. [CrossRef] [PubMed]

9. Wan, Y.; Schwaninger, H.; He, P.; Wang, Y. Comparison of resistance to powdery mildew and downy mildew in Chinese wild grapes. Vitis 2007, 46, 132-136.

10. Merdinoglu, D.; Wiedemann-Merdinoglu, S.; Coste, P.; Dumas, V.; Haetty, S.; Butterlin, G.; Greif, C.; Adam-Blondon, A.; Bouquet, A.; Pauquet, J. Genetic analysis of downy mildew resistance derived from Muscadinia rotundifolia. Acta Hortic. 2003, 603, 451-456. [CrossRef] 
11. Fischer, B.M.; Salakhutdinov, I.; Akkurt, M.; Eibach, R.; Edwards, K.J.; Töpfer, R.; Zyprian, E.M. Quantitative trait locus analysis of fungal disease resistance factors on a molecular map of grapevine. Theor. Appl. Genet. 2004, 108, 501-515. [CrossRef] [PubMed]

12. Welter, L.J.; Göktrk-Baydar, N.; Akkurt, M.; Maul, E.; Eibach, R.; Töpfer, R.; Zyprian, E.M. Genetic mapping and localization of quantitative trait loci affecting fungal disease resistance and leaf morphology in grapevine (Vitis vinifera L). Mol. Breed. 2007, 20, 359-374. [CrossRef]

13. Bellin, D.; Peressotti, E.; Merdinoglu, D.; Wiedemann-Merdinoglu, S.; Adam-Blondon, A.-F.; Cipriani, G.; Morgante, M.; Testolin, R.; di Gaspero, G. Resistance to Plasmopara viticola in grapevine 'Bianca' is controlled by a major dominant gene causing localized necrosis at the infection site. Theor. Appl. Genet. 2009, 120, 163-176. [CrossRef] [PubMed]

14. Marguerit, E.; Boury, C.; Manicki, A.; Donnart, M.; Butterlin, G.; Némorin, A.; Wiedemann-Merdinoglu, S.; Merdinoglu, D.; Ollat, N.; Decroocq, S. Genetic dissection of sex determinism, inflorescence morphology and downy mildew resistance in grapevine. Theor. Appl. Genet. 2009, 118, 1261-1278. [CrossRef] [PubMed]

15. Zyprian, E.; Welter, L.J.; Akkurt, M.; Ebert, S.; Salakhutdinov, I.; Göktürk-Baydar, N.; Eibach, R.; Töpfer, R. Genetic analysis of fungal disease resistance in grapevine. Acta Hortic. 2009, 827, 535-538. [CrossRef]

16. Moreira, F.M.; Madini, A.; Marino, R.; Zulini, L.; Stefanini, M.; Velasco, R.; Kozma, P.; Grando, M.S. Genetic linkage maps of two interspecific grape crosses (Vitis spp.) used to localize quantitative trait loci for downy mildew resistance. Tree Genet. Genom. 2011, 7, 153-167. [CrossRef]

17. Schwander, F.; Eibach, R.; Fechter, I.; Hausmann, L.; Zyprian, E.; Töpfer, R. Rpv10: A new locus from the Asian Vitis gene pool for pyramiding downy mildew resistance loci in grapevine. Theor. Appl. Genet. 2012, 124, 163-176. [CrossRef] [PubMed]

18. Gururani, M.A.; Venkatesh, J.; Upadhyaya, C.P.; Nookaraju, A.; Pandey, S.K.; Park, S.W. Plant disease resistance genes: Current status and future directions. Physiol. Mol. Plant Pathol. 2012, 78, 51-65. [CrossRef]

19. Nürnberger, T.; Brunner, F.; Kemmerling, B.; Piater, L. Innate immunity in plants and animals: Striking similarities and obvious differences. Immunol. Rev. 2004, 198, 249-266. [CrossRef] [PubMed]

20. Ron, M.; Avni, A. The receptor for the fungal elicitor ethylene-inducing xylanase is a member of a resistance-like gene family in tomato. Plant Cell 2004, 16, 1604-1615. [CrossRef] [PubMed]

21. Schwessinger, B.Z.C. News from the frontline: Recent insights into PAMP-triggered immunity in plants. Curr. Opin. Plant Biol. 2008, 11, 389-395. [CrossRef] [PubMed]

22. Van der Biezen, E.A.; Jones, J.D. Plant disease-resistance proteins and the gene-for-gene concept. Trend. Biochem. Sci. 1998, 23, 454-456. [CrossRef]

23. Xiao, S.; Ellwood, S.; Calis, O.; Patrick, E.; Li, T.; Coleman, M.; Turner, J.G. Broad-spectrum mildew resistance in Arabidopsis thaliana mediated by RPW8. Science 2001, 291, 118-120. [CrossRef] [PubMed]

24. Wang, W.; Wen, Y.; Berkey, R.; Xiao, S. Specific targeting of the Arabidopsis resistance protein RPW8.2 to the interfacial membrane encasing the fungal haustorium renders broad-spectrum resistance to powdery mildew. Plant Cell 2009, 21, 2898-2913. [CrossRef] [PubMed]

25. Eckardt, N.A. The "Arabidopsis" RPW8 resistance protein is recruited to the extrahaustorial membrane of biotrophic powdery mildew fungi. Plant Cell 2009, 21, 2543. [CrossRef] [PubMed]

26. Wang, W.; Devoto, A.; Turner, J.G.; Xiao, S. Expression of the membrane-associated resistance protein RPW8 enhances basal defense against biotrophic pathogens. MPMI 2007, 20, 966-976. [CrossRef] [PubMed]

27. Li, Q.; Li, J.; Sun, J.L.; Ma, X.F.; Wang, T.T.; Berkey, R.; Yang, H.; Niu, Y.Z.; Fan, J.; Li, Y.; et al. Multiple evolutionary events involved in maintaining homologs of resistance to powdery mildew 8 in Brassica napus. Front. Plant Sci. 2016, 7. [CrossRef] [PubMed]

28. Sun, J.L. Polymorphism Analysis and Functional Identification of RPW8 Homologues in Brassica napus. Master's Thesis, China Agricultural University, Beijing, China, June 2014.

29. Henanff, G.L.; Heitz, T.; Mestre, P.; Mutterer, J.; Walter, B.; Chong, J. Characterization of Vitis vinifera NPR1 homologs involved in the regulation of pathogenesis-related gene expression. BMC Plant Biol. 2009, 9, 54-67. [CrossRef] [PubMed]

30. Kortekamp, A. Expression analysis of defence-related genes in grapevine leaves after inoculation with a host and a non-host pathogen. Plant Physiol. Biochem. 2006, 44, 58-67. [CrossRef] [PubMed]

31. Wu, J.; Zhang, Y.; Zhang, H.; Huang, H.; Folta, K.M.; Lu, J. Whole genome wide expression profiles of Vitis amurensis grape responding to downy mildew by using Solexa sequencing technology. BMC Plant Biol. 2010, 10, 234. [CrossRef] [PubMed] 
32. Gu, K.; Yang, B.; Tian, D.; Wu, L.; Wang, D.; Sreekala, C.; Yang, F.; Chu, Z.; Wang, G.L.; White, F.F. R gene expression induced by a type-III effector triggers disease resistance in rice. Nature 2005, 435, 1122-1125. [CrossRef] [PubMed]

33. Kortekamp, A.; Welter, L.; Vogt, S.; Knoll, A.; Schwander, F.; Töpfer, R.; Zyprian, E. Identification, isolation and characterization of a CC-NBS-LRR candidate disease resistance gene family in grapevine. Mol. Breed. 2008, 22, 421-432. [CrossRef]

34. Radwan, O.; Gandhi, S.; Heesacker, A.; Whitaker, B.; Taylor, C.; Plocik, A.; Kesseli, R.; Kozik, A.; Michelmore, R.W.; Knapp, S.J. Genetic diversity and genomic distribution of homologs encoding NBS-LRR disease resistance proteins in sunflower. Mol. Genet. Genom. 2008, 280, 111-125. [CrossRef] [PubMed]

35. Li, X.; Wu, J.; Yin, L.; Zhang, Y.; Qu, J.; Lu, J. Comparative transcriptome analysis reveals defense-related genes and pathways against downy mildew in Vitis amurensis grapevine. Plant Physiol. Biochem. 2015, 95, 1-14. [CrossRef] [PubMed]

36. Xiao, S.Y.; Charoenwattana, P.; Holcombe, L.; Turner, J.G. The Arabidopsis genes RPW8.1 and RPW8.2 confer induced resistance to powdery mildew diseases in tobacco. MPMI 2003, 16, 289-294. [CrossRef] [PubMed]

37. Monteiro, F.; Sebastiana, M.; Pais, M.S.; Figueiredo, A. Reference gene selection and validation for the early responses to downy mildew infection in susceptible and resistant Vitis vinifera cultivars. PLoS ONE 2013, 8, e72998. [CrossRef] [PubMed]

38. Livak, K.J.; Schmittgen, T.D. Analysis of relative gene expression data using real-time quantitative PCR and the $2^{-\Delta \Delta C t}$ method. Methods 2001, 25, 402-408. [CrossRef] [PubMed]

39. Xiang, J.; Li, X.; Wu, J.; Yin, L.; Zhang, Y.; Lu, J. Studying the mechanism of Plasmopara viticola RxLR effectors on suppressing plant immunity. Front. Microbiol. 2016, 7, 709. [CrossRef] [PubMed]

40. Gallois, P.; Marinho, P. Leaf disk transformation using Agrobacterium tumefaciens-expression of heterologous genes in tobacco. Method Mol. Biol. 1995, 49, 39-48.

41. Li, X.; Zhang, Y.; Yin, L.; Lu, J. Overexpression of pathogen-induced grapevine TIR-NB-LRR gene VaRGA1, enhances disease resistance and drought and salt tolerance in Nicotiana benthamiana. Protoplasma 2016, 254, 1-13. [CrossRef] [PubMed]

42. Jiang, M.; Zhang, J. Effect of abscisic acid on active oxygen species, antioxidative defence system and oxidative damage in leaves of maize seedlings. Plant Cell Physiol. 2001, 42, 1265-1273. [CrossRef] [PubMed]

43. Zhang, D.Z.; Wang, P.H.; Zhao, H.X. Determination of the content of free proline in wheat leaves. Plant Physiol. Commun. 1990, 4, 62-65. 